\title{
Study on morphological characters of some mangrove plants in South-eastern Ayeyarwady Delta of Myanmar
}

\begin{abstract}
A study on the mangrvoe plants in Pyapon Township, Ayeyarwady Region, Southeastern Ayeyarwady Delta (between Lat. $94^{\circ} 30^{\prime}$ and $95^{\circ} 45^{\prime}$ North and between Long. $15^{\circ} 30^{\prime}$ and $16^{\circ} 25^{\prime}$ East), Myanmar was conducted within the period of March 2016 to February 2017. A total of 18 species of mangroves plants were recorded in the natural mangrove areas. In the present study, the taxonomic descriptions of mangrove plants were presented.
\end{abstract}

Keywords: taxonomic, Ayeyarwady Delta
Volume 8 Issue 4 - 2019

\author{
Kyi Kyi Myint, Myat Myat Nwe, Tin Lay Mar \\ Department of Botany, Magway University, Myanmar
}

Correspondence: Kyi Kyi Myint, Associate Professor, Department of Botany, Magway University, Myanmar, Email Kyikyimyintbotany@gmail.com

Received: July 06, 2019 | Published: July 25, 2019

\section{Introduction}

'Mangrove' has been variously defined in literature. The oxford dictionary mentioned the words 'mangrove' and 'mangrowe' since 1613 , indicating tropical trees or shrubs found in coastal swamps with tangled roots that grow above the ground, whereas the Americans, the Spanish, and the Portuguese used the term 'Mangle' and 'Mangue' indicating trees and shrubs of the genus Rhizophora. ${ }^{1}$ Later, the term 'mangrove' was referred to the individual plant or tidal forest or both, as 'Mangrove plants' and 'Mangrove ecosystem'.2 In addition, mangroves have been defined by Hamilton and Snedaker ${ }^{3}$ as salt tolerant ecosystems of the intertidal regions along coastlines. Moreover, the term 'mangrove' is used to define both the plants that occur in tidal forests, and to describe the community itself., Mangrove plants mostly grow within the sheltered intertidal flat deltaic lands, funnel-shaped bays, broad estuarine mouths, shallow or frequently tidal inundated coast lines. ${ }^{6}$

Mangrove forests were of crucial significance for local people, providing food, shelter and, medicinal and other uses. The local people depend on mangroves for fire-wood, charcoal, timber, poles and many other purposes. Traditional uses of mangrove resources currently continue side by side with large-scale and intensive exploitation using high-capital investment and technologies such as charcoal production and firewood. In recent years, efforts to convert mangrove land for other uses, such as fish- or shrimp-ponds or industrial and human settlements have been increasing in number and size. Nevertheless, mangrove provided directly and indirectly uses in various ways.

The mangrove forests provide livelihood and employment to wood cutters, fishermen, honey and wax collector, shell collectors, timber traders and their workers and other peoples who work both seasonally and round the year in the forest.

Mangroves occur in 112 countries and global coverage has been estimated at over $179,000 \mathrm{~km}^{2},{ }^{7} 10$ million hectares, ${ }^{8}$ 14-15million hectares ${ }^{9}$ and 24 million hectares,${ }^{10}$ more than 18 million ha. ${ }^{4}$ A study by Giri et al. ${ }^{11}$ estimated that the total mangrove forest area of the world in 2000 was $137,760 \mathrm{~km}^{2}$ with the largest extent of mangroves found in Asia (42\%) followed by Africa (20\%), North and Central America $(15 \%)$, Oceania (12\%) and South America (11\%). The Sundarbans, the mangrove forest in Bangladesh, is the largest mangrove forest in the world. Australia has the third largest area of mangroves in the world. India is one of the 15 most mangrove-rich countries having 2.7 $\%$ of global total area of mangroves. Asia contains most of the world's mangroves with $46 \% .{ }^{12}$ The largest areas of mangrove in Southeast Asia are found in Indonesia (almost $60 \%$ Southeast Asia's total), Malaysia (11.7\%), Myanmar (8.8\%), Papua New Guinea (8.7\%) and Thailand (5.0\%).

Globally, there are 110 recognized species of plants classified as mangroves, belonging to 20 different families. ${ }^{13}$ However, Tomlinson ${ }^{4}$ reported that around 34 major and 20 minor mangrove species belonging to about 20 genera in over 11 families have been recorded globally.

It is well known that Southeast Asia's mangroves are the best developed and probably the most species-diverse in the world. Giesen \& Wulffraat, 1998 reported that 52 species are found in the Southeast Asian. Wang et al. ${ }^{14}$ reported that 59 species in 41 genera and 29 families of mangrove plants were recorded in India and 26 species of 12 families and 15 genera were from China. Hening ${ }^{15}$ recorded 69 species under 34 families in the whole of Sundarban mangrove. However, Karim ${ }^{16}$ reported 123 plant species belonging to 22 families representing 30 genera in Bangladesh. Santisuk ${ }^{17}$ reported that 74 species under 53 genera were recorded in Thailand.

Myanmar is endowed with a rich diversity of habitat types arising largely from its unusual ecological diversity. Myanmar has more than 2,000kilometres of coastline along the Bay of Bengal. Generally, it comprises the Rakhine, Ayeyarwady Delta and Tanintharyi region, where mangroves are common. About 8.8percent of Southeast Asia's mangroves are located in Myanmar, of which 46 percent is located in Ayeyarwady (Irrawaddy) Division, 37 percent in Tanintharyi Division and 17 percent in Rakhine State. The largest mangrove areas were observed in Ayeyarwady Delta having 180,826 ha compared to other areas of Rakhine (64,777ha) and Tanintharyi (140,485ha). Mangrove forests are mainly found in Bogalay, Pyapon and Laputta townships, which are situated in the south of the Ayeyarwady Delta. ${ }^{18}$ The total area was about 530,000 hectares in 1980 , but it decreased to 425,000 hectares by 2000 , or perhaps as low as 382,032 ha. 
The various authors studied the marphological characters of mangrove plants along the various localities of Myanmar coastal regions byThan Htay and Saw Han, ${ }^{19}$ Aung Myint \& Kyaw Soe, ${ }^{20}$ Cherry Aung, ${ }^{21}$ San Tha Tun, ${ }^{22}$ Tin Hla and Htun Paw Oo, ${ }^{23}$ Thi Thi Htaik, ${ }^{24}$ Mar Lar, ${ }^{25}$ San Tha Tun, ${ }^{26}$ Nyo Nyo San. ${ }^{27}$ The purpose of this study is to elucidate the distribution, occurrence and taxonomic descriptions of mangroves.

\section{Materials and methods}

Study area: The natural mangrove formations were observed along the Tebinzeik creek and Thaung-ga-done creek that located between Htaung Gyi Tan and Ah Shey Hpyar villages and War Kone village. War Kone village is situated at the inner part of Ahmar Sub-Township. These villages were located the south-eastern Ayeyarwady Delta, Ayeyarwady Region, Pyapon Township (between latitudes $94^{\circ} 30^{\prime}$ and $95^{\circ} 45^{\prime}$ North and between longitudes $15^{\circ} 30^{\prime}$ and $16^{\circ} 25^{\prime}$ East), Myanmar.

Observation of Mangrove plants in the field: In this study, mangrove plants were collected from March 2016 to February 2017. The morphology of mangroves were recorded from the natural mangrove areas. Taxonomic descriptions were described in the fieldwork and floral parts such as flowers, leaves were taken to the laboratory to make herbarium sheets and preserved.To study the morphology of the mangroves, twenty transects were assigned perpendicular to the creek crossing through the forest from seaward to landward. Permanent plots $(10 \mathrm{~m} \times 10 \mathrm{~m})$ were set along each transect. $\mathrm{T}_{1}-\mathrm{T}_{7}$ were set from near the Htaung Gyi Tan village, $T_{8}-T_{16}$ from Ah Shey Hpyar and $T_{17}-$ $\mathrm{T}_{20}$ from War Gone villages respectively. The location of the transect lines along the creek of mangrove areas were shown in the Figure 1.

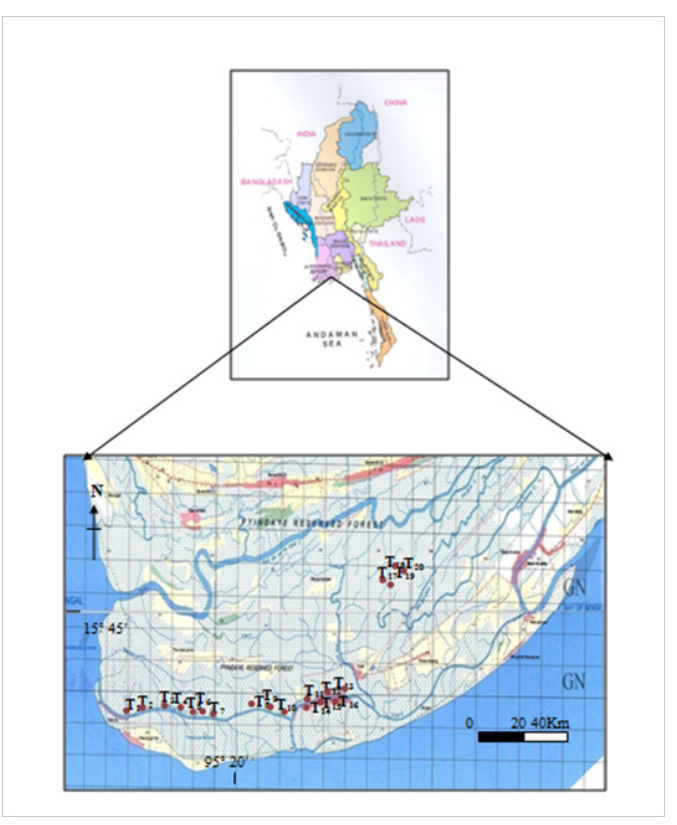

Figure I Map showing the study areas within the South-eastern Ayeyarwady Delta, Pyapon Township, Ayeyarwady Region, Myanmar

\section{Results}

In the present study18 species of mangroves that belong to 11 families and 13 genera have been recorded from the natural mangrove areas of Htaung Gyi Tan, Ah Shey Hpyar and War Gone villages, Pyapon Township, South-eastern Ayeyarwady Delta. The recorded plants included 14 species of woody trees, 2 species of shrubs, 1 species of monocot plams and 1 species of fern.

The classified list of mangrvoe plants were mentioned in Table 1 and the morphological descriptions were also presented.

\section{Acanthus volubilis Vahl.}

\section{Syn: Dilivaria ebracteata Vers.}

Shrubs, upto $1.5 \mathrm{~m}$ height. Stem solid, glabrous, terete. Leaves simple, opposite and decussate, exstipulate; petiole $0.5-0.7 \mathrm{~cm}$ long and $0.3-0.4 \mathrm{~cm}$ broad, solid, glabrous, pulvinous; lamina oblong to elliptic-oblong, $10.0-12.0 \mathrm{~cm}$ long and $4.0-5.0 \mathrm{~cm}$ broad, the base angled, the margin spiny to very spiny and wavy, the tip acute.
Inflorescence raceme, spike $11.0-16.0 \mathrm{~cm}$ long, peduncled. Flowers 5.5-6.0 $\mathrm{cm}$ long and $3.3-3.5 \mathrm{~cm}$ across; bract $1,1.5-1.8 \mathrm{~cm}$ long and 0.8 $1.0 \mathrm{~cm}$ broad, curved, ovoid, entire, acute, basal cup-shaped, glabrous, greenish yellow; 2 bracteoles partly covered by bract, smaller than bract; showy flower, erect, sessile, bisexual, zygomorphic, complete, hypogynous. Sepals 4, aposepalous, 2 outer one larger, 2 inner one smaller, ovate, $1.7-2.0 \mathrm{~cm}$ long and $0.8-1.1 \mathrm{~cm}$ broad, acute, basal curved, coriaceous, glabrous, greenish, persistent. Petals 1, ovate, 3.5$4.0 \mathrm{~cm}$ long and $2.5-3.0 \mathrm{~cm}$ broad, entire, obtuse, coriaceous, slightly fleshy, slightly curved, margin outer folded, large, showy, white, deciduous. Stamens 4, free, filament about $1.9 \mathrm{~cm}$ long and about $0.25 \mathrm{~cm}$ broad, fleshy, solid, white, glabrous, flattened, erect; anther dithecous, about $0.8 \mathrm{~cm}$ long, basifixed, densely white, pubescent, longitudinal dehiscence, inserted, introse. Carpels 2, syncarpous, ovary dumble shaped, $0.2-0.3 \mathrm{~cm}$ long, glabrous, terete, two locules, two ovules in each locule, ovary superior, axile placentation, style terminal, persistent, glabrous, white, stigma bifid. Fruit capsule, 2.5$3.0 \mathrm{~cm}$ long and $0.8-1.0 \mathrm{~cm}$ in diameter, glabrous. Seeds $0.7-1.0 \mathrm{~cm}$ long, kidney shaped, grey (Figure 2). 
Study on morphological characters of some mangrove plants in South-eastern Ayeyarwady Delta of

Table I Classified list of mangrove plants

\begin{tabular}{|c|c|c|c|c|c|c|c|c|}
\hline & Division & Class & Order & Family & Genus & Species & Local name & Type \\
\hline I & Magnoliphyta & Magnoliopsida & Scorphulariales & Acanthaceae & Acanthus & Acanthus volubilis & Khayar-phyu & Shrub \\
\hline 2 & & Liliopsida & Arecales & Arecaceae & Nypa & Nypa fruticans & Dani & Palm \\
\hline 3 & & Magnoliopsida & Lamiales & Avicenniaceae & Avicennia & Avicennia marina & $\begin{array}{l}\text { Thame-phyu,Thame- } \\
\text { ywet-leit }\end{array}$ & Tree \\
\hline 4 & & & & & & Avicennia officinalis & $\begin{array}{l}\text { Thame-gyi, Thame- } \\
\text { net, Thame-ywet- } \\
\text { wyne }\end{array}$ & Tree \\
\hline 5 & & & Euphorbiales & Euphorbiaceae & Excoecaria & Excoecaria agallocha & Tayaw, Thayaw & Tree \\
\hline 6 & & & Sapindales & Meliaceae & Xylocarpus & Xylocarpus moluccensis & Kyana, Kyat-nan & Tree \\
\hline 7 & & & Primulales & Myrsinaceae & Aegiceras & Aegiceras corniculatum & Yae-kha-yar & Tree \\
\hline 8 & & & Plumbaginales & Plumbaginaceae & Aegialitis & Aegialitis rotundifolia & $\begin{array}{l}\text { Pinle-sar, Sar-thar- } \\
\text { pin }\end{array}$ & Shrub \\
\hline 9 & Polypodiophyta & Polypodiopsida & Pteridiales & Pteridaceae & Acrostichum & Acrostichum aureum & Hnget-gyi-taung & Fern \\
\hline 10 & Magnoliphyta & Magnoliopsida & Rhizophorales & Rhizophoraceae & Bruguiera & Bruguiera cylindrica & Hnan-byu & Tree \\
\hline II & & & & & & Bruguiera gymnorrhiza & $\begin{array}{l}\text { Byu-oak-saung, } \\
\text { Byu-ku-lar }\end{array}$ & Tree \\
\hline 12 & & & & & & Bruguiera sexangula & Byu-shwe-war & Tree \\
\hline 13 & & & & & Ceriops & Ceriops tagal & Madama-myaw & Tree \\
\hline 14 & & & & & Rhizophora & Rhizophora apiculata & $\begin{array}{l}\text { Byu-chidauk-ahpho, } \\
\text { Byu-chidauk-ywet- } \\
\text { chun }\end{array}$ & Tree \\
\hline 15 & & & Myrtales & Sonneratiaceae & Sonneratia & Sonneratia alba & Lame & Tree \\
\hline 16 & & & & & & Sonneratia apetala & Kambala & Tree \\
\hline 17 & & & & & & Sonneratia griffithii & Laba & Tree \\
\hline 18 & & & Malvales & Sterculiaceae & Heritiera & Heritiera fomes & $\begin{array}{l}\text { Pinle-kanazo, Yae- } \\
\text { kanazo }\end{array}$ & Tree \\
\hline
\end{tabular}
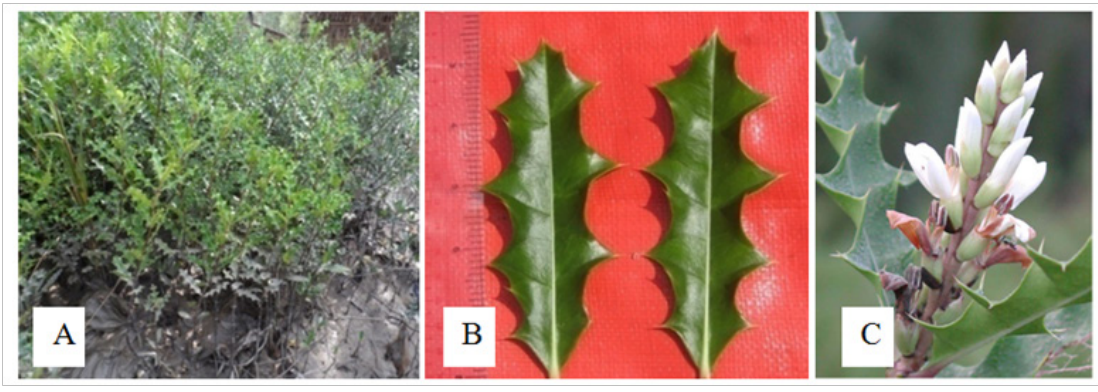

Figure 2 Acanthus volubilis: (A) Habit. (B) Leaves and (C) Inflorescences 2. Nypa fruticans Wurmb. I78I. 
Flowering and fruting periods- May to August

\section{Nypa fruticans}

Palms, 3.5-4.5m height, dichotomously branched, rhizomatous stems. Leaves spirally arranged, pinnately compound, upto $5 \mathrm{~m}$ long, shiny, glabrous, green, rachis stout, sheathing base. Inflorescence spadix on a long peduncle, upto $50.0 \mathrm{~cm}$ long, several spathe enclosed flowers, peduncle erect, stout, glabrous, green. Male flowers gregariously on each peduncle, covered by spathe; tepals 6 , apotepalous in two whorls, ovate elliptic, upto $0.8 \mathrm{~cm}$ long and $0.5 \mathrm{~cm}$ broad, entire, acute, tufted, glabrous, shiny; stamens 3, united to form central column, anthers dithecous, upto $0.4 \mathrm{~cm}$ long. Female flowers enclosed by spathe, tepals 6 ; capels 3 , funnel shaped, upto $1.0 \mathrm{~cm}$ height, angular, unilocular, single ovule, basal placentation, style and stigma 0 . Fruit globose. Seeds broadly ovoid (Figure 3 ).

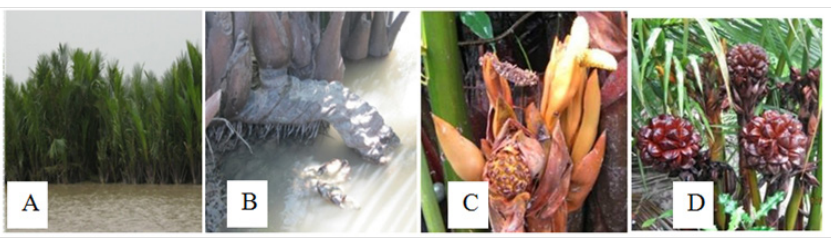

Figure 3 Nypa fruticans: (A) Habit. (B) Rhizome. (C) Male and female inflorescence and (D) Fruits

Flowering and fruting periods- November to July

Avicennia marina (Forsk.) Vierh. 1907.

Syn: Sceura marina Forsk.1775.

Rach bruce Trav. 1790.

\section{Racka torrid J.F.Gmel.1791.}

Halodendron thourasi Roem. \& Schult. 1818.

Racka ovate Roem. \& Shult. In L. 1818.

Trees, up to $10 \mathrm{~m}$ height; pencil-like pneumatophores, arising upward from the horizontal spreading roots. Leaves opposite and decussate, exstipulate, simple; petiole $1.0-1.3 \mathrm{~cm}$ long, slightly flattened, glabrous, pulvinous; lamina ovate-lanceolate, $8.0-10.0 \mathrm{~cm}$ long and $2.5-3.5 \mathrm{~cm}$ broad, glabrous, the base cuneate or tapering, the margin entire, the tip acute, coriaceous, the upper surface shinning and the lower surface much silvery or white, pubescent. Inflorescence condensed, terminal or axillary cyme, globose, compound spike, infrequently branched, glabrous, peduncle long, up to $10 \mathrm{~cm}$. Flowers $0.5-0.9 \mathrm{~cm}$ long and $0.4-0.5 \mathrm{~cm}$ across, sessile, orange yellow, bracteate, bracteolate, complete, regular, hypogynous. Sepals 4 , aposepalous, elliptic, $0.25-0.35 \mathrm{~cm}$ long and $0.15-0.2 \mathrm{~cm}$ broad, entire, blunt, coriaceous, persistent, outer surface hairy, tomentose, imbricate. Petals 4, synpetalous, the tube short, th lobe 4, ovate, 0.3 $0.4 \mathrm{~cm}$ long, $0.25-0.3 \mathrm{~cm}$ wide, acute, orange yellow, hairy outside and glabrous within, valvate. Stamens 4 , about $0.2 \mathrm{~cm}$ long, epipetalous, filament round, white, glabrous; anther dithecous, yelloe, basifixed, longitudinal dehiscence, extrorse, exserted. Carpel 3, ovary $0.2-0.3 \mathrm{~cm}$ long, oblong, style terminal, short, stigma 2, bi-fid, glabrous below, hairy above. Fruit capsule, compressed, shortly straight beak (Figure 4).

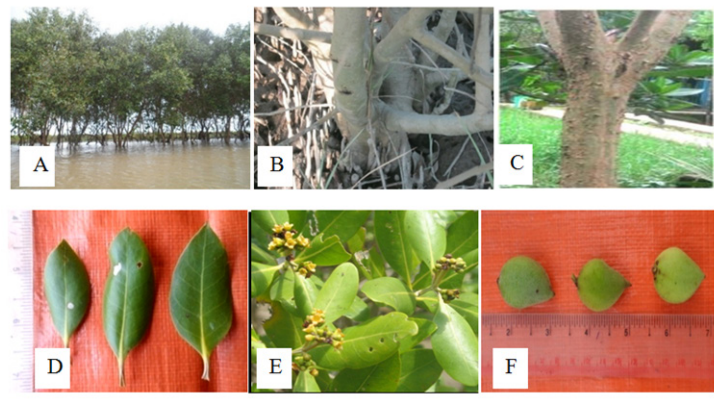

Figure 4 Avicennia marina: (A) Habit. (B) Stem base and its pneumatophores (C) Stem. (D) Leaves (E) Inflorescence and (F) Fruits.

Flowering and fruting periods- March to October

Avicennia officinalis L.1753.

Syn: Anacardium orientale Houst.1662.

Oepata rheed Hort.1683.

Avicennia tomemtosa Willd. 1800.

Avicennia oepata Hamilt. 1835.

Medium to tall trees, $15-20 \mathrm{~m}$ height; pneumatophores numerous, long, spongy, pencil like erect. Leaves opposite and decussate, simple, exstipulate; petiole $1.3-1.5 \mathrm{~cm}$; lamina obovate-oblong, $9.5-10.5 \mathrm{~cm}$ long and $5.0-5.8 \mathrm{~cm}$ broad, the margin entire, obtuse or rounded apex, green, leathery not much silvery beneath, glabrous. Inflorescence compound spike, with 10-12 flowers in each branch, capitate globose, panicles, up to $7.8 \mathrm{~cm}$ long, peduncle up to $24.0 \mathrm{~cm}$ long. Flowers $0.4-1.1 \mathrm{~cm}$ long and $1.1-1.5 \mathrm{~cm}$ broad at anthesis; bracts 2 , small with fimbriate margin; bracteoles 2 ; sessile, complete, bisexual, dull yellow, actinomorphic, hypogynous. Sepals 4, aposepalous, elliptic, $0.5-0.6 \mathrm{~cm}$ long and $0.25-0.3 \mathrm{~cm}$ broad, entire, acute, coriaceous, pubescent, quincuncial, persistent. Petals 4, apopetalous, elliptic, $0.5-0.8 \mathrm{~cm}$ long and $0.5-0.6 \mathrm{~cm}$ broad, entire, acute, curved in the tips, quincuncial, orange yellow, deciduous. Stamens 4, apostemonous, alternate, $0.3-0.4 \mathrm{~cm}$ long, filaments $0.2-0.25 \mathrm{~cm}$ long, round, glabrous; anther dithecous, $0.1-0.15 \mathrm{~cm}$ long, yellow, basifixed, extrorse, exserted, longitudinal dehiscent. Carpel elliptic or slightly oblique, $0.65-0.7 \mathrm{~cm}$ long, with shortly hairs, 1 locule, style $1,0.15-0.75 \mathrm{~cm}$ long, glabrous, stigma capitate. Fruit fleshy, ovoid, $3.5-5.0 \mathrm{~cm}$ long and $2.4-2.8 \mathrm{~cm}$ broad, densely hairy, flattened, wrinkled at the base and with a short narrow beak. Seed single, dark green (Figure 5).
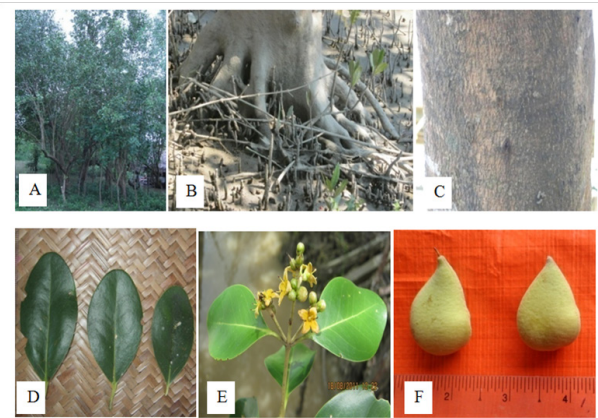

Figure 5 Avicennia officinalis: (A) Habit. (B) Stem base and its pneumatophores. (C) Stem. (D) Leaves. (E) Inflorescence and (F) Fruits. 
Flowering and fruting periods- March to October

Excoecaria agallocha L.1759.

Syn: E. camettia Willd.

\section{E. affinis Endl.}

\section{Stillingia agallocha Baill.}

Medium trees, up to $15.0 \mathrm{~m}$ height, with poisonous milky latex; root without any aerial growth. Stems round, solid. Leaves spirally arranged, simple, exstipulate, occasionally reddish; petiole $1.5-2.0 \mathrm{~cm}$ in length and 3.0-3.5 $\mathrm{cm}$ broad, glabrous; lamina ovate elliptic, 5.0$7.0 \mathrm{~cm}$ in length and $2.5-3.5 \mathrm{~cm}$ broad. Male inflorescence catkins, peduncle $5.0-7.0 \mathrm{~cm}$ long, deciduous, bracteate, sessile, dioecious, small, odorless; tepals 3 , ovate, $0.12-0.15 \mathrm{~cm}$ long, apotepalous, irregularly acute, yellow, small, valvate, persistent; stamens 3 , free, filament $1.2-1.3 \mathrm{~cm}$ long, glabrous, anther dithecous, about $0.1 \mathrm{~cm}$ long, basixed, extrorse, exserted, longitudinal dehiscence. Female inflorescence mixed cyme, bracteate, glandular, sessile, bracteoles pair, round, glabrous, dioecious; tepals 3, ovate, apotepalous, valvate, green, glabrous, acute, small, persistent, odorless; Carpel 3 , syncarpous, superior, ovary ovoid, 3 locule, basal placentation, one ovule in each locule, stlyes 3 , curved, simple, about $0.2 \mathrm{~cm}$ long, stigma 3 , terminally bent, slightly pubescent. Fruits 3 -lobed, schizocarp, about $0.6 \mathrm{~cm}$ in diameter, dehiscing into 3 cocci to release the solitary seeds. Seeds black, about $6.0 \mathrm{~mm}$ in diameter (Figure 6).
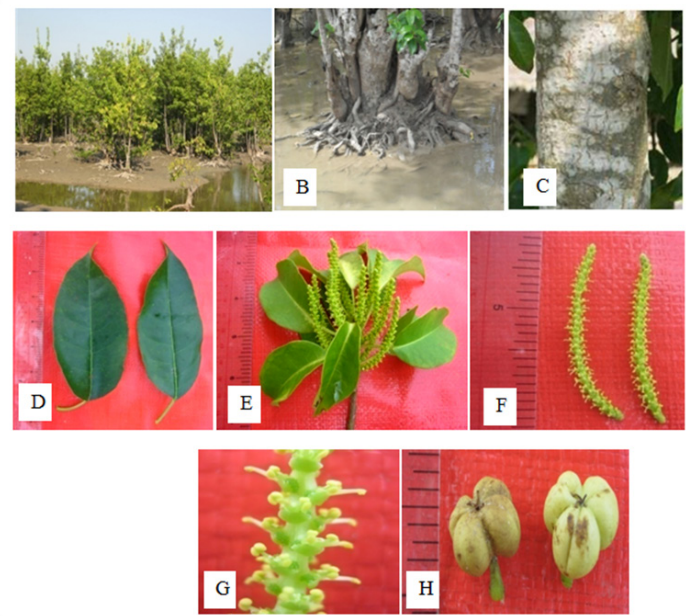

Figure 6 Excoecaria agallocha: (A) Habit. (B) Stem base. (C) Stem. (D) Leaves. (E) Male inflorescence. (F) Male flowers. (G) Female flowers and $(\mathrm{H})$ Fruit.

Flowering and fruting periods - May to August

Xylocarpus moluccensis (Lamk.) M. Roem.1879.

Syn: Carapa molluccensis Lam.

C. indica Juss.

Medium trees, up to $10 \mathrm{~m}$ height, buttresses absent, conical like pneumatophores. Stems rough, erect, solid, woody, longitudinal fissured. Leaves paripinnately compound, leaflets $4-6$, exstipulate; petiole $0.5-0.7 \mathrm{~cm}$ long, dark brown, glabrous, pulvinous; lamina ovate-elliptic, $10.0-13.5 \mathrm{~cm}$ long and 5.0-7.0 $\mathrm{cm}$ width, entire, slightly mucronate, upper surface dark green, lower surface whitish, glabrous, coriaceous. Inflorescence united cymes, $7.0-7.5 \mathrm{~cm}$ long, with distinct main axis. Flowers tetramerous, 0.6-
$0.65 \mathrm{~cm}$ long and about $0.3 \mathrm{~cm}$ across; pedicel about $0.5 \mathrm{~cm}$ long, glabrous, brown. Sepal 4, aposepalous, ovate, about $0.15 \mathrm{~cm}$ long and about $0.2 \mathrm{~cm}$ broad, green, blunt, entire, coriaceous, glabrous, valvate, deciduous. Petal 4, apopetalous, ovate, about $0.3 \mathrm{~cm}$ long and about $0.25 \mathrm{~cm}$ broad, thin, white, slightly curved, odourless, twisted, deciduous. Stamens 8 , fuse to form globose tube, staminal tube present, 8 apical short lobe; lobe ovate, about $0.15 \mathrm{~cm}$ long; anther dithecous, inserted, introrse, dorsifixed, longitudinal dehiscence, inferior. Carpel 8, syncarpous; ovary globose, about $0.2 \mathrm{~cm}$ in diameter; 8-locules, two ovules in each locule, axile placentation, style terminal, about $0.1 \mathrm{~cm}$ long, gradually tapering, glabrous; stigma 1, flat. Fruits round spherical or globose, with long woody stalk, fruit coad woody, pendulous and hanging on branches, $12.0-15.0 \mathrm{~cm}$ across, 4 sutures of woody valves, well filled with $15-20$ seeds. Seeds angular look like pyramidal, tetrahedral, hard (Figure 7).

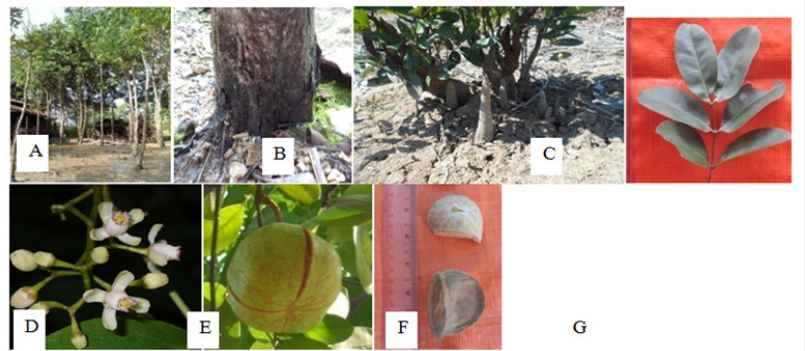

Figure 7 Xylocarpus moluccensis: (A) Habit. (B) Stem base. (C) Pneumatophores. (D) Leaves. (E) Inflorescence. (F) Fruit and $(G)$ Seeds

Flowering and fruting periods-March to September

Aegiceras corniculatum (L.) Blanco.1837.

Syn: Rhizophora corniculatum L. 1754.

Aegiceras majus Gaertn. 1788.

Small tree, up to $4 \mathrm{~m}$ height. Stems solid, cylindrical. Leaves alternate, simple, exstipulate, petiole $0.8-0.9 \mathrm{~cm}$ long, $0.2-0.3 \mathrm{~cm}$ width, glabrous, pulvinous; lamina obovate to oblong, $8.4-8.7 \mathrm{~cm}$ length and $4.0-4.2 \mathrm{~cm}$ broad, the base cuneate, the margin entire, the tip round to emerginate, shiny, coriaceous. Inflorescence simple umbel, terminal. Flowers $1.2-1.4 \mathrm{~cm}$ long and $0.8-1.0 \mathrm{~cm}$ in across; bracts very minute, deciduous, triangle in shaped; pedicel $0.6-0.7 \mathrm{~cm}$ long and $0.1 \mathrm{~cm}$ width, glabrous; ebracteolate, complete, bisexual, regular, actinomorphic, pentamerous, twisted, hypogynous, fragrance. Sepals 5, aposepalous, $0.4 \mathrm{~cm}$ long and $0.3 \mathrm{~cm}$ broad, round apex, curved, coriaceous, twisted, sepaloid, persistent, inferior. Petals 5, synpetalous, about $1.0 \mathrm{~cm}$ long and $1.0-1.2 \mathrm{~cm}$ broad, white, reflexed, entire, acuminate, twisted. Stamens 5, opposite to petal, filaments $0.4 \mathrm{~cm}$ long, base united to form a tube, anthers dithecous, sagittate, creamy white, exserted, introrse, dorsifixed, longitudinal dehiscence, inferior. Carpel 1, monocarpelly, about $0.3 \mathrm{~cm}$ long, 2 ovules in each locule, free central placentation, style 1 , terminal, about $0.7 \mathrm{~cm}$ long, little red sports in the middle of style, glabrous, soft, stigma absent, superior. Fruits capsule, $6.0-8.0 \mathrm{~cm}$ long, completely curved with pointed apex, calyx persistent at the base. One seeded, $1.0-1.1 \mathrm{~cm}$ long (Figure 8).

Flowering and fruting periods- January to September

Aegialitis rotundifolia Roxb.1824.

Syn: A.annulata Kz. 


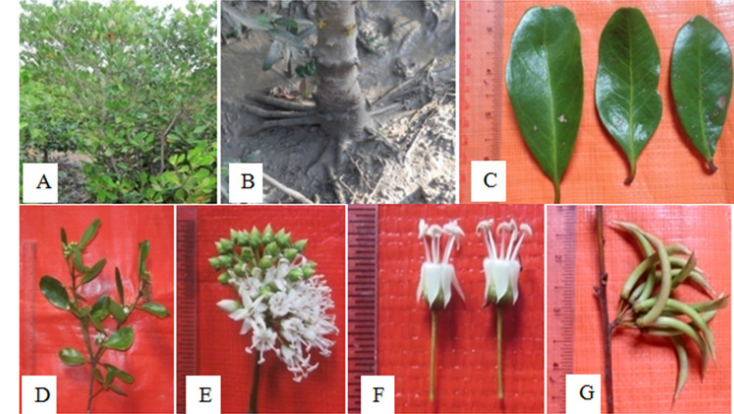

Figure 8 Aegiceras corniculatum: (A) Habit. (B) Stembase. (C) Leaves (D) Inflorescence $(E)$ Close up view of inflorescence. $(F)$ Flowers and $(G)$ Fruits .

Shrubs, up to $3 \mathrm{~m}$ height, without any aerial roots, latex present, mucilage. Stems cylindrical, base swollen look like buttresses, branches more or less spongy with leaf scars. Leaves alternate, simple, exstipulate, petioles $9.0-10.0 \mathrm{~cm}$ long and $0.5-0.7 \mathrm{~cm}$ in diameter, tubular leaf-sheath, glabrous; lamina broadly ovate, $9.0-9.2 \mathrm{~cm}$ long and $7.0-7.5 \mathrm{~cm}$ broad, the upper surface shiny, slightly fleshy, dark green, the base cuneate, the margin entire, the tip obtuse. Infloresecence raceme, peduncle $6.5-7.0 \mathrm{~cm}$ long, developed from sheathing petiole. Flowers $1.5-1.8 \mathrm{~cm}$ long and about $1.0 \mathrm{~cm}$ in across; bract ovatelanceolate, $1.0-1.2 \mathrm{~cm}$ long and $0.5-0.7 \mathrm{~cm}$ broad; pedicel $0.3-0.4 \mathrm{~cm}$ long, terete; bracteoles $0.6-0.8 \mathrm{~cm}$ long, entire, obtuse, mucronate apex, curved, green; complete, bisexual, regular, actinomorphic, pentamerous, hypogynous. Clyx 5, synsepalous, slightly connate at base, lobe $1.0 \mathrm{~cm}$ long and $0.2 \mathrm{~cm}$ broad, entire, acute, glabrous, coriaceous, green, valvate. Petals 5, apopetalous, linear oblong, 11.0$12 \mathrm{~cm}$ long and $0.15-0.2 \mathrm{~cm}$ wide, entire, obtuse, thin, white, glabrous, odourless, imbricate. Stamens 5, slightly connate at the base, short stamina tube, $0.3-0.5 \mathrm{~cm}$ long; filament about $1.3 \mathrm{~cm}$ long, equal in length, exserted, white, glabrous; anther dithecous, sagittate, about $0.2 \mathrm{~cm}$ long, yellowish, basifixed, longitudinal dehiscence, extrose. Carpel 5, syncarpous, ovary oblong, about $0.3 \mathrm{~cm}$ long, one locule, basal placentation, style 5 , terminal, about $0.4 \mathrm{~cm}$ long, glabrous, white. Fruit capsule, $6.0-8.0 \mathrm{~cm}$ long, with persistent calyx, linear. Seeds $6.0-7.0 \mathrm{~cm}$ long, black (Figure 9).

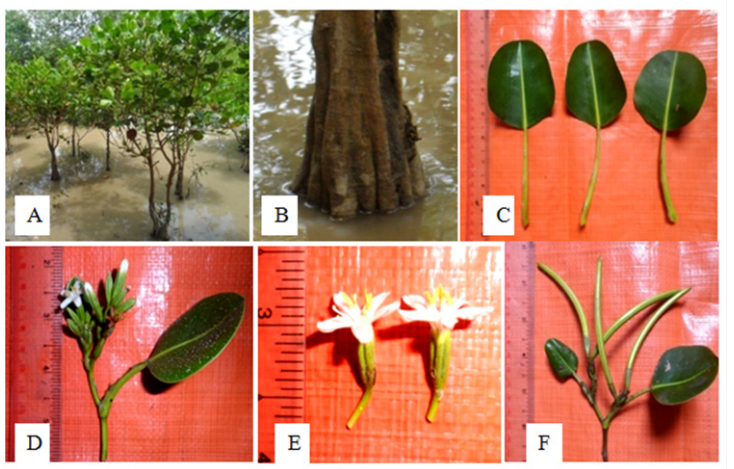

Figure 9 Aegialitis rotundifolia: (A) Habit. (B) Stem base. (C) Leaves. (D) Inflorescence. (E) Flowers and (F) Fruits.

Flowering and fruting periods - April to October

Acrostichum aureum L.

Erect fern, attaining 1-2m in height, small bushy appearance; rhizomatous with fibrous like roots. Frond simple, stout, erect, $1.0 \mathrm{~m}$ long and $4.0 \mathrm{~cm}$ width, covered with large scales. Tips of fertile frond rusty-brown, maturing dark brown during spore release, tips of sterile frond blunt with a short tip, unicostate reticulate venation, glabrous, coriaceous. Mature frond become sporophyllous, diffuse sporangia at abaxial surface, mixed sporangia on both mid vein, brown sporangia stalked, upper globose (Figure 10).

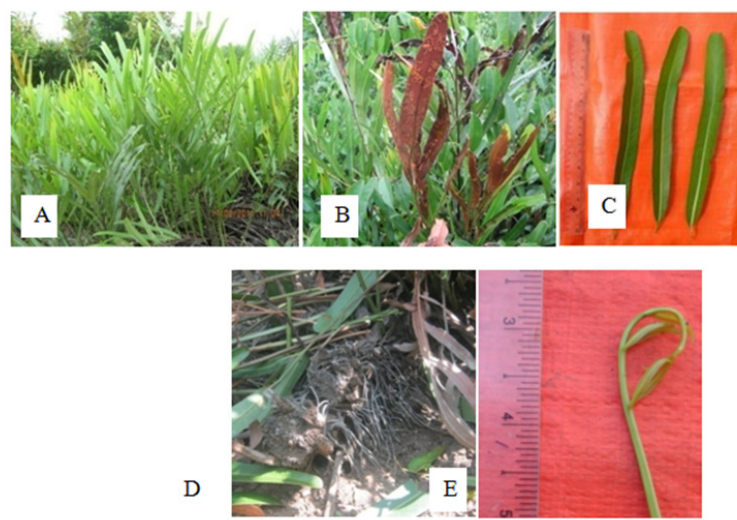

Figure 10 Acrostichum aureum: (A) Habit. (B) Fertile fronds. (C) Leaves. (D) Rhizome and (E) Circinate young fronds.

Sporangia formation - November to February

Bruguiea cylindrica (L.) Blume. 1827.

Syn: Rhizophora cylindrica L.1753.

Rhizophora caryophylloides Burm. f. 1768.

Bruguiera caryophylloides Blume, Enum.1827.

Large tree, upto $20.0 \mathrm{~m}$ height; pneumatophores knee like and buttresses forms. Stem glabrous, solid. Leaves simple, opposite and decussate, exstipulate, petiole $2.0-2.3 \mathrm{~cm}$ long, pulvinous; lamina ovate-lanceolate, elliptic, $13.8-14.0 \mathrm{~cm}$ long and $4.8-5.2 \mathrm{~cm}$ broad, the margin entire, the tip acute, glabrous, coriaceous. Inflorescence cymose, 3-flowers in each peduncle, peduncle long, terete, green, glabrous. Flowers $1.0-1.3 \mathrm{~cm}$ long and $0.3-0.5 \mathrm{~cm}$ in across, ebracteate, pedicel $0.2-0.3 \mathrm{~cm}$ long, glabrous, smooth, pale green, ebracteolate, complete, bisexual, regular, actinomorphic, epigynous, creamy white in colour. Sepals 8 , synsepalous, $0.4-0.5 \mathrm{~cm}$ long and $0.2 \mathrm{~cm}$ broad, surface smooth, pointed apex, entire, valvate, thick, hard, persistent. Petals 8 , aposepalous, $0.35-0.4 \mathrm{~cm}$ long and $0.15-0.2 \mathrm{~cm}$ broad, each petal folding, two parts, thin, terminal small ciliated and basal hairy along the margin. Stamens16, free, 8 in groups, 2 stamens in each petal, filaments unequal, about $0.15-0.3 \mathrm{~cm}$ long, introrse, inserted, anther dithecous, $0.15-0.2 \mathrm{~cm}$ long, basifixed, longitudinal dehiscence. Pistils cup-shaped, ovary $0.15-0.2 \mathrm{~cm}$ long, $2-4$ celled, one ovule in each locule, axile placentation, style terminal, $0.75-0.9 \mathrm{~cm}$ long, glabrous, stigmas bifid, hairy. Fruit capsule, pendulous, persistent calyx, the lobes bent towards the pedicels (Figure 11).

Flowering and fruting periods- March to November

Bruguiera gymnorrhiza (L.) Lamk.1797-98.

Syn: B. conjugate (L.) Merr.

B. rheedii Miq. 

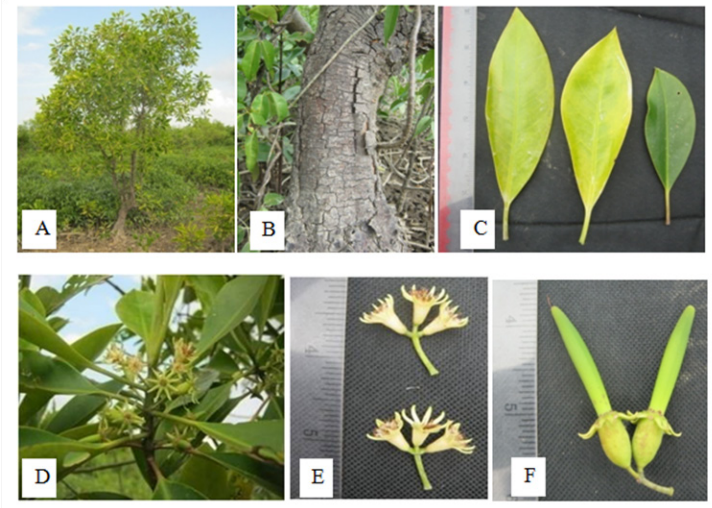

Figure I I Bruguiea cylindrica:(A) Habit.(B) Stem. (C) Leaves.(D) Inflorescences. (E) Flowers and (F) Fruits.

\section{Rhizophora gymnorhiza Roxb.}

Everygreen tree, up to $10 \mathrm{~m}$ height; aerial roots from knee like pneumatophores developing from bending horizontal underground roots; root buttress thickened the trunk base. Stem solid. Leaves opposite and decussate, simple; petiole $3.5-4.5 \mathrm{~cm}$ long, glabrous, reddish, solid, pulvinous; lamina broadly ovate-lanceolate, 16.0$19.0 \mathrm{~cm}$ long and $5.0-6.5 \mathrm{~cm}$ broad, reddish distinct mid-vein in the leaves, the margin entire, the tip cute, coriaceous, shiny, glabrous. Inflorescence axillary, solitary, large, cymose. Flower pendulous, elliptical, $3.0-3.5 \mathrm{~cm}$ long and $2.5-3.5 \mathrm{~cm}$ broad at anthesis, ebracteate; pedicel about $2.0 \mathrm{~cm}$ long, round, glabrous, reddish; ebracteolate, complete, bisexual, regular, actinomorphic, cyclic, epigynous. Sepals 13-16, aposepalous, about $1.3 \mathrm{~cm}$ long, with distinct ribbed, reddish, conspicuously fleshy lobes, apicular, apex pointed, hard, persistent, valvate. Petals 13-16, apopetalous, about $1.0 \mathrm{~cm}$ long and about $0.2 \mathrm{~cm}$ broad, oblong, coriaceous, reddish, upper surface slippery, terminally 4 cilia, white, $0.3-0.4 \mathrm{~cm}$ long. Stamens 26-32, free, two stamens pair within one petal, pair stamens with unequal filament; filament up to $0.3 \mathrm{~cm}$ long, glabrous; anther about $0.25 \mathrm{~cm}$ long, dithecous, basifixed, longitudinal dehiscence, introrse, inserted. Pistil cup-shaped, 2-4 locules, 2 ovules in each locule, style terminal, about $0.7 \mathrm{~cm}$ long, glabrous, stigma 3-lobed, glabrous. Fruit capsule, pendulous with persistent calyx, one-seeded (Figure 12).

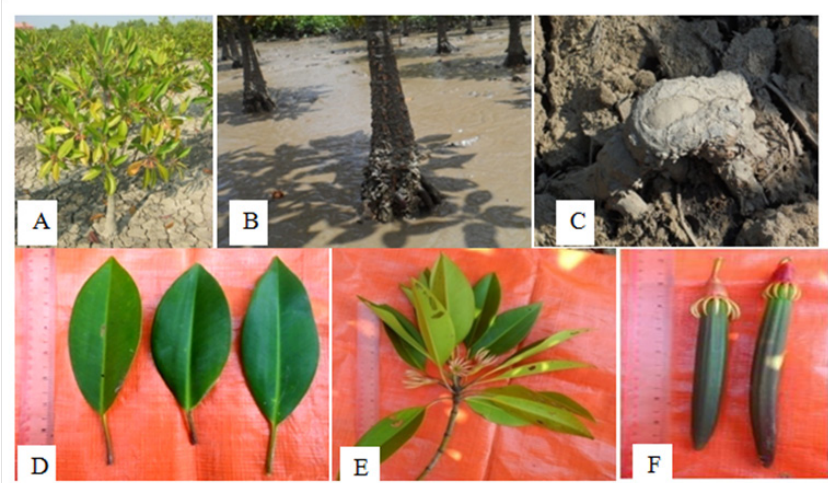

Figure I2 Bruguiera gymnorrhiza: (A) Habit. (B) Stem. (C) Knee root. (D) Leaves. (E) Inflorescences and (F) Fruits.

Flowering and fruting periods- March to October
Bruguiera sexangula (Lour.) Poir. Lamk. 1816.

Syn: B. eriopetala W. \& A.

B. rumphii $\mathrm{B} 1$.

B. parietosa Griff.

Small trees, up to 5-10m height; aerial roots from root buttresses and developed significantly the conical trunk base or broom like trunk with stilt roots base when young and knee like pneumatophores. Stems solid. Leaves opposite and decussate, simple; petiole $2.8-3.0 \mathrm{~cm}$ long, glabrous, greenish yellow, solid, pulvinous; lamina ovate-lanceolate, $11.5-13.5 \mathrm{~cm}$ long and $4.5-5.6 \mathrm{~cm}$ broad, glabrous, yellowish distinct mid-vein, the upper surface shiny, the margin entire, the tip acute, coriaceous, fleshy, stipulate. Inflorescence axillary, solitary, large, cymose. Flower large, pendulous, $3.8-4.0 \mathrm{~cm}$ long, ebracteate; pedicel 0.8-0.9cm long, curved, terete, solid, smooth, glabrous, yellowish, pendulous, ebracteolate, complete, bisexual, regular, actinomorphic, cyclic, epigynous. Sepals 10-12, synsepalous, sepal tube $1.5 \mathrm{~cm}$ long, sepal lobe $2 \mathrm{~cm}$ long, with distinct ribbed, yellowish, conspicuously fleshy lobes, apicular, apex pointed, hard, persistent, valvate. Petals $10-12$, apopetalous, $0.7-0.8 \mathrm{~cm}$ long, bilobed, folding into one, each lobe $0.18-0.2 \mathrm{~cm}$ broad, lanceolate, terminally not ciliated, coriaceous, tufted, basal side pubecent, reddish brown, glabrous, outer surface slippery, pale red inner surface, slightly rought. Stamens $24-26$, free, 10-12 groups, 2 stamens into each folding petal, unequal filaments, up to $0.7 \mathrm{~cm}$ long, the base of filament hairy, white, inserted, introrse; anther dithecous, $0.38-0.4 \mathrm{~cm}$ long, basifixed, longitudinal dehiscence, yellow. Pistil cup-shaped, ovary $0.4-0.5 \mathrm{~cm}$ long, $2-4$ celled, 2 ovules in each locule, axile placentation, style terminal, $0.8-1.0 \mathrm{~cm}$ long, white, glabrous, stigma 3, small, ovate, glabrous, entire, fleshy. Fruits indehiscent pod, pendulous, green, slightly angular (Figure13).
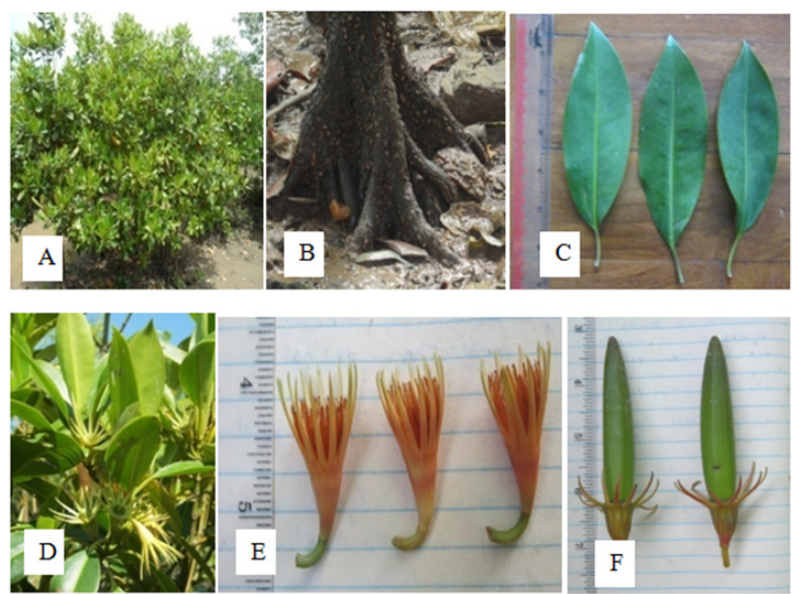

Figure I3 Bruguiera sexangula: (A) Habit. (B) Stem base. (C) Leaves. (D) Inflorescences. (E) Flowers and (F) Fruits.

Flowering and fruting periods - March to October

Ceriops tagal (Perr.) C. B. Robison. 1908.

Syn: Rhizophora tagal Perr. 1824.

Ceripos candolleana Arn., Ann.1838.

Small to moderate trees, $5.0-8.0 \mathrm{~m}$ height; aerial roots broom like and form buttresses on trunk base. Stems solid. Leaves opposite and decussate, simple; petiole $1.8-2.0 \mathrm{~cm}$ long and $0.2 \mathrm{~cm}$ broad, glabrous, 
flattened, pulvinous; lamina elliptic, $9-10 \mathrm{~cm}$ long and $5.8 \mathrm{~cm}$ broad, the base obtuse, the margin entire, apex rounded, coriaceous, glabrous. Inflorescence cymose, axillary, flowers cluster, on long slender peduncle, peduncle $0.9-1.0 \mathrm{~cm}$ long, glabrous, flattened. Flowers pentamerous, $0.5-0.6 \mathrm{~cm}$ long and $0.4-0.45 \mathrm{~cm}$ in diameter, bisexual, complete, regular; pedicel short, $0.1 \mathrm{~cm}$ long, glabrous, minute. Sepals 5 , aposepalous, elliptic-lanceolate, $0.4-0.45 \mathrm{~cm}$ long and $0.2-0.25 \mathrm{~cm}$ broad, entire, thick, green, acute, persistent. Petals 5, apopetalous, oblong, $0.25-0.3 \mathrm{~cm}$ long, entire, thin, reddish brown, thick, terminally 3 -clavate appendages present, valvate. Stamens 10 , free, filament $0.25-0.28 \mathrm{~cm}$ long, unequal, 5 short and 5 long, reddish brown; anthers dithecous, sagittate, $0.1 \mathrm{~cm}$ long, shorter than the filament length, light brown, dorsifixed, introrse, inserted, longitudinally dehiscence. Carpel 3, syncarpous, inferior, elliptic globose, $0.15-0.18 \mathrm{~cm}$ long, axile placentation, 2 locules, 2 ovules in each locule, style terminal, $0.2-0.3 \mathrm{~cm}$ long, reddish brown, glabrous, stigma 3 , shortly tri-fid. Fruits indehiscent, $20-26 \mathrm{~cm}$ long, with 5 persistent calyx, green to yellowish brown, surface warty, ridged and grooved, hanging and pointed. Seed single (Figure 14).
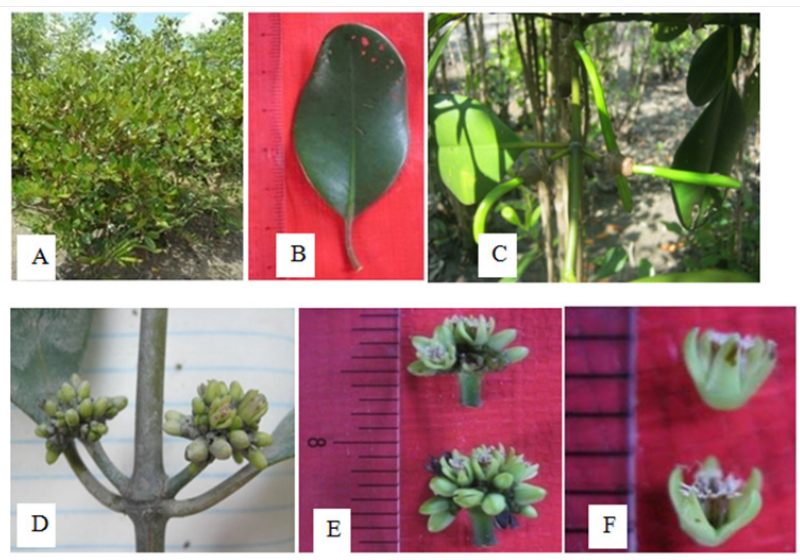

Figure I4 Ceriops tagal: (A) Habit. (B) Leaf. (C) Fruit. (D) Inflorescence as seen. (E) Inflorensence and (F) Flower.

Flowering and fruting periods- February to September

Rhizophora apiculata Blume. 1827.

Syn: Rhizophora candellaria DC. 1828.

Rhizophora conjugate Sensu. Arn. 1859.

Medium trees, up to $12 \mathrm{~m}$ height. Silt roots, looping from lower branches and trunk base. Stem solid, glabrous; bark grey to dark grey, longitudinally fissured. Leaves opposite and decussate, simple, exstipulate; petiole $2.6-2.8 \mathrm{~cm}$ long and $0.35-0.4 \mathrm{~cm}$ broad, slightly flattened, glabrous, green, pulvinous; lamina ovate-elliptic, 14.0$14.3 \mathrm{~cm}$ long and $6.4-7.0 \mathrm{~cm}$ broad, the margin entire, the tip acute, coriaceous. Inflorescence cymose, $2.5-2.9 \mathrm{~cm}$ long, 2 -flowered on each peduncle, peduncle $0.8-1.0 \mathrm{~cm}$ long and $0.5-0.7 \mathrm{~cm}$ diameter, glabrous. Flowers $1.2-1.4 \mathrm{~cm}$ long, ebracteate, sessile, complete, bisexual, regular, actinomorphic, glabrous, complete, regular, odorless, globose in bud, medium. Sepals 4, aposepalous, ovate, $1.2-1.6 \mathrm{~cm}$ long, $0.9-1.0 \mathrm{~cm}$ broad, entire, acute, fleshy, thick, glabrous, valvate, persistent. Petals 4, apopetalous, lanceolate, $0.8-1.0 \mathrm{~cm}$ long and 0.2$0.25 \mathrm{~cm}$ broad, entire, acute, glabrous, fleshy, deciduous, odourless. Stamens 11-12, free, sessile, anther dithecous, sagittate, $1.0-1.2 \mathrm{~cm}$ long, introrse, inserted, longitudinal dehiscence, white. Carpels 2, syncarpous, ovary globose, $0.2-0.25 \mathrm{~cm}$ long, glabrous, 2 locules, 2 ovules in each locule, axile placentation, style terminal, very short, stigma bifurcate. Fruit capsule, $45.0-47.0 \mathrm{~cm}$ long and $1.5-2.0 \mathrm{~cm}$ in diameter, tapering towards the apex, with 4 persistent sepals. Seeds 1, $1.5-1.7 \mathrm{~cm}$ long and $2.0 \mathrm{~cm}$ in dameter (Figure 15).
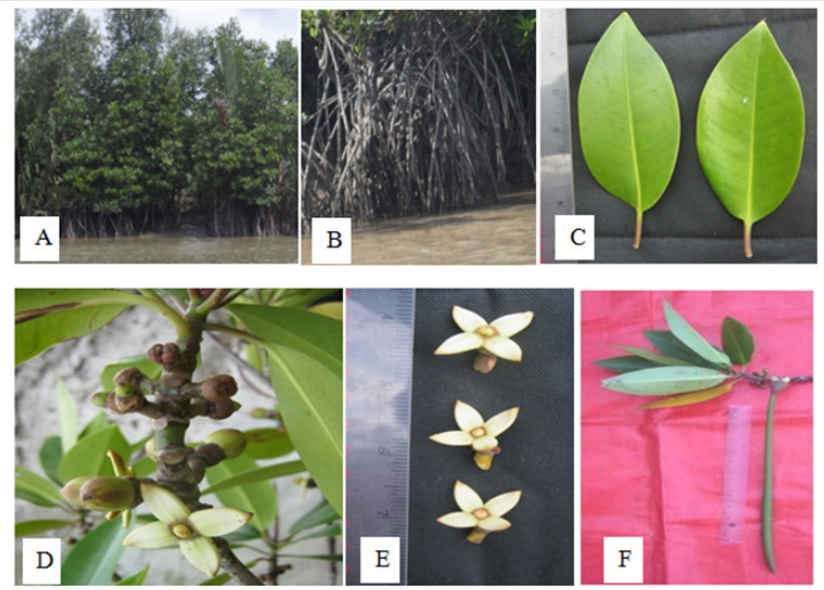

Figure I5 Rhizophora apiculata: (A) Habit. (B) Stilt roots. (C) Leaves. (D) Inflorescences. (E) Flowers and (F) Fruit.

Flowering and fruting periods- March to September

Sonneratia alba J. Smith. 1879.

Syn: S. mossambicensis Klotz.

S. acida Benth.

Tree, up to $10 \mathrm{~m}$ height; bark pale brown to deep brown, smooth with fine longitudinal fissures; pencil like pneumatophores. Leaves simple, opposite and decussate, exstipulate; petiole $0.5-1.0 \mathrm{~cm}$ long; lamina obovate-elliptic to almost round, $7.5-8.0 \mathrm{~cm}$ long and 4.0 $5.0 \mathrm{~cm}$ broad, margin entire, the tip blunt, glabrous, coriaceous, fleshy. Inflorescence terminal, few flowered cyme. Flowers $6.5-7.0 \mathrm{~cm}$ and $7.0-8.5 \mathrm{~cm}$ in across at anthesis, ebracteate, pedicel $4.0-5.5 \mathrm{~cm}$ long, glabrous, complete, bisexual, regular, actinomorphic, perigynous. Calyx 6, synsepalous, the tube cup-shaped, with 6 lobes, oblongelliptic, $2.5-4.0 \mathrm{~cm}$ long and $1.0-1.5 \mathrm{~cm}$ broad, green outside and red or purple inside, linear, entire, acute, glabrous, fleshy, coriaceous, valvate, persistent. Petals 6 , polypetalous, linear, upto $3.5 \mathrm{~cm}$ long and upto $0.5 \mathrm{~cm}$ broad, truncate, entire, acuminate, white, glabrous, valvate, deciduous, glabrous. Stamens numerous, free, showy, filament $4.0-4.5 \mathrm{~cm}$ long, white, glabrous; anther dithecous, $0.1 \mathrm{~cm}$ in diameter, yellow, dorsifixed, exserted, extrorse, longitudinal dehiscence. Carpel 6 , syncarpous, ovary cup-shaped, $2.0-2.5 \mathrm{~cm}$ long and $4.0-4.5 \mathrm{~cm}$ in diameter, shining, flattened, round, 20 locules, numerous ovules in each locule, axile placentation, style terminal, $5.0-6.0 \mathrm{~cm}$ long, glabrous, persistent,stigma capitate, $0.5-0.2 \mathrm{~cm}$ in diameter, glabrous. Fruits globose-berry, $5.0-8.0 \mathrm{~cm}$ in diameter, lower part somewhat enclosed in turbinate calyx, at maturity flat and with sharp pointed, long style. Seeds numerous (Figure 16).

Flowering and fruting periods-April to October

Sonneratia apetala Buch. 1800

Syn: Kambala apetala Rafin. Sylb. Tell. 1838.

Blatti apetala O. K. Rev. Gen. 1891. 

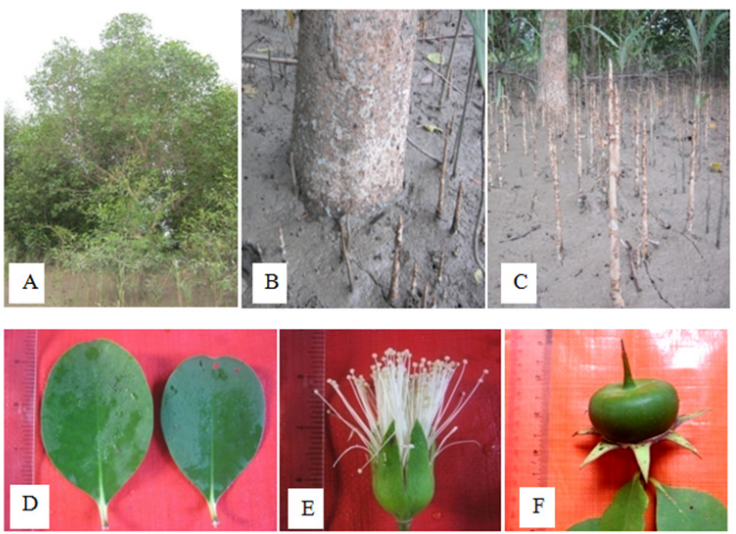

Figure I 6 Sonneratia alba; (A) Habit. (B) Stem base. (C) Pneumatophores. (D) Leaves. (E) Flower and (F) Fruit.

Trees, up to $20 \mathrm{~m}$ height, drooping twings and branchlets; pencil like pneumatophores present, tapering the ends. Stem irregularly fissured. Leaves opposite and decussate, simple, exstipulate; petiole about $0.6 \mathrm{~cm}$ long, glabrous, pulvinous; lamina oblong-lanceolate, narrow, gradually tapering towards the apex, $8.0-12.0 \mathrm{~cm}$ long and $2.0-3.0 \mathrm{~cm}$ wide, glabrous, shiny, the base attenuated, the margin entire, the tip obtuse. Inflorescences terminal or axillary, mostly 3 flowered, dichasial cyme. Flowers $1.7-3.0 \mathrm{~cm}$ long and $2.5-3.0 \mathrm{~cm}$ in across, bracterate; pedicel $1.8-2.0 \mathrm{~cm}$ long; complete, bisexual, regular, actinomorphic, tetramerous, hypogynous, yellowish white. Calyx 4, synsepalous, the tube cup-shped, the lobes elliptic, $1.5-1.7 \mathrm{~cm}$ long and $0.7-0.8 \mathrm{~cm}$ broad, entire, acute, valvate, persistent. Petals absent. Stamens many, free, filament $1.0 \mathrm{~cm}$ long, creamy yellowish white, glabrous, inserted, anther dithecous, pale yellow, $1.0 \mathrm{~cm}$ long, dorsifixed, longitudinally dehiscence. Carpel 4 , syncarpous, ovary dumble shaped, $0.4-0.5 \mathrm{~cm}$ long and $0.6-0.8 \mathrm{~cm}$ in diameter, superior, 5 locules, numerous ovules in each locule, axile placentation, style 1 ,terminal, glabrous, yellowish white, $1.5-2.0 \mathrm{~cm}$ long, curved, stigma 1 , lage and umbrella-shaped. Fruits globose, berry, $2.0-2.2 \mathrm{~cm}$ in diameter, depressed, arranged on the flattened persistent calyx. Seeds $1.4 \mathrm{~cm}$ long, brown, triangular, ridged (Figure 17).
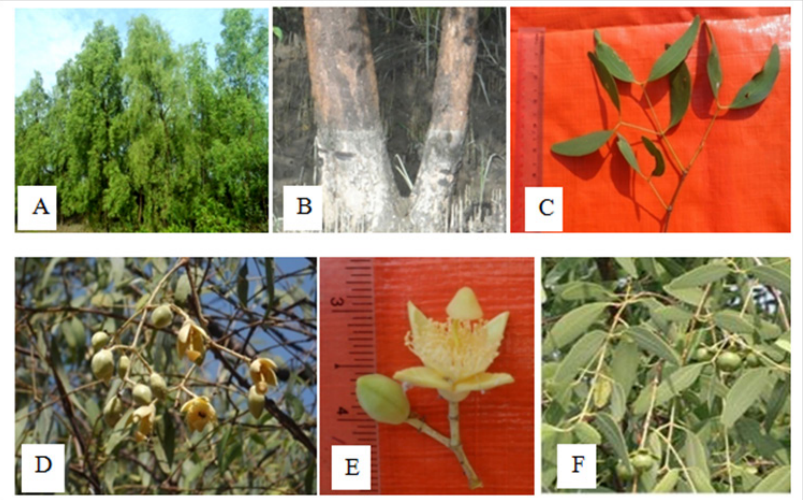

Figure 17 Sonneratia apetala: (A) Habit (B) Stem base and its pneumatophores. (C) Leaves. (D) Inflorescence as seen. (E) Flower and (F) Fruits.

Flowering and fruting periods - June to October17. Sonneratia griffithii Kurz. 1871.

\section{Sonneratia griffithii Kurz. 1871}

Syn: Sonneratiaalba Griff.
Tree, upto $15 \mathrm{~m}$ height; pencil like pneumatophores with tapering end. Stem terete, glabrous, solid; bark cream to brown, with longitudinal fissures. Leaves simple, opposite and decussate, leathery and exstipulate; petiole $0.1-0.3 \mathrm{~cm}$ long, glabrous, pulvinous; lamina obovate or broadly oval, $13.0-13.3 \mathrm{~cm}$ long and $11.0-11.3 \mathrm{~cm}$ wide, the base very broadly round or subcordate, the margin entire, the tip round, fleshy, glabrous, shiny. Inflorescence terminal, cymose. Flowers 3.0$4.5 \mathrm{~cm}$ long and $5.0-6.8 \mathrm{~cm}$ across, ebracteate, pedicel upto $1.0 \mathrm{~cm}$ long, glabrous, ebracteolate, incomplete, bisexual, actinomorphic and perigynous. Sepals 6 , synsepalous, the tubes cup-shaped, the lobes lanceolate-elliptic, $2.0-4.0 \mathrm{~cm}$ long and $0.5-1.0 \mathrm{~cm}$ wide, entire, acute, sepaloid, fleshy, slightly flattened, valvate, persistent. Petals absent. Stamens numerous, free, filaments $2.0-3.5 \mathrm{~cm}$ long, white, soon shed following anthesis, glabrous; anther dithecous, basifixed, exserted, white, introrse, longitudinal dehiscence. Carpals 6 , syncarpous, ovary globose, $2.0-3.0 \mathrm{~cm}$ long and $4.0-5.0 \mathrm{~cm}$ in diameter, green, glabrous, multilocular, numerous ovules in each locule, axile placentation; style 1, terminal, $3.0-4.0 \mathrm{~cm}$ long, soft, terete, glabrous, persistent; stigma 1 , globose. Fruit is globose berry, $5.0-7.0 \mathrm{~cm}$ in diameter, terminally into short style beak, partly enclosed by peristent green sepals at base. Seeds numerous, angular, light brown (Figure 18).
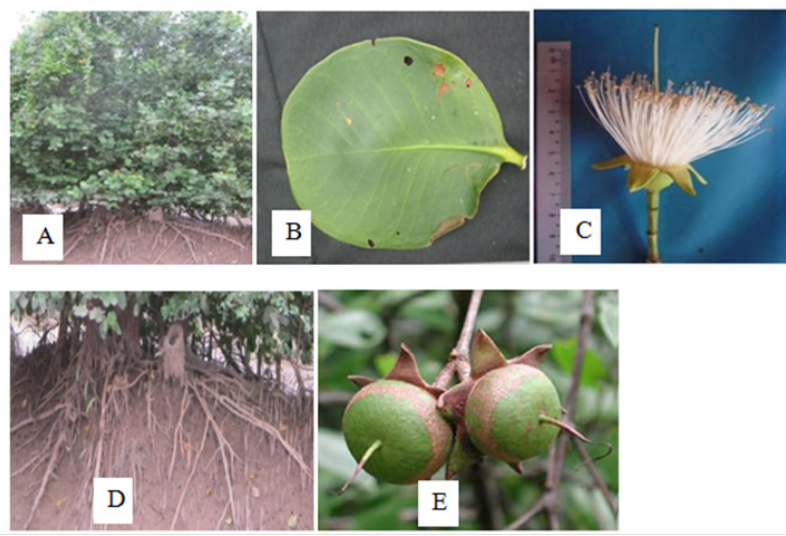

Figure 18 Sonneratia griffithii: (A) Habit. (B) Leaf. (C) Flower. (D) Pneumatophores. and (E) Fruits.

Flowering and fruting periods-April to October

Heritiera fomes Bunch. Ham.1800.

Syn: Heritiera minor Roxb.1832.

Every green trees, up to $10 \mathrm{~m}$ height; stem base buttress, blunt end peg-like pneumatophores present. Leaves alternate, simple, stipules in pair in each node; petioles about $1.0 \mathrm{~cm}$ long, terete, grey, slightly pulvinous; lamina elliptic-lanceolate, $8.0-14 \mathrm{~cm}$ long and $3.0-4.5 \mathrm{~cm}$ wide, the margin entire, the tip acute, tapering, coriaceous, slightly hard, dorsal surface pale green, glabrous, ventral surface whitish grey, rough. Inflorescence mixed; peduncle $5.5-6.0 \mathrm{~cm}$ long, bent and pendulous, much branched. Flowers $1.0 \mathrm{~cm}$ long and $0.5 \mathrm{~cm}$ across, small, ebracterate, regular, unisexual, odourless; pedicel about $0.5 \mathrm{~cm}$ long, pubescent, soft. Calyx $4-5$, synsepalous, calyx tube cupshaped, $0.3-0.35 \mathrm{~cm}$ long, outer surface orange-yellow, inner surface reddish-orange towards the base, imbricate, inferior. Petals absent. Male flowers: stamens 5, fused to form pistillode; anther encircling the apical portion of the pistillode, to form globose, dithecous with apical white beak, yellow, longitudinal dehiscence, introrse, inserted. Female flowers: carpels loosely attached, $0.2-0.25 \mathrm{~cm}$ long, each ovary flattened, globose, pubescent, one ovule in each locule, basal placentation; style terminal, about $0.1 \mathrm{~cm}$ long, white; stigma pentafid. 
Fruit capsule, sub-globose, $1.5-2.0 \mathrm{~cm}$ long, up to $5.0 \mathrm{~cm}$ in diameter, pericarp brown, hard, woody divided into 2 halves by distinct ridged, 1-seeded (Figure 19).
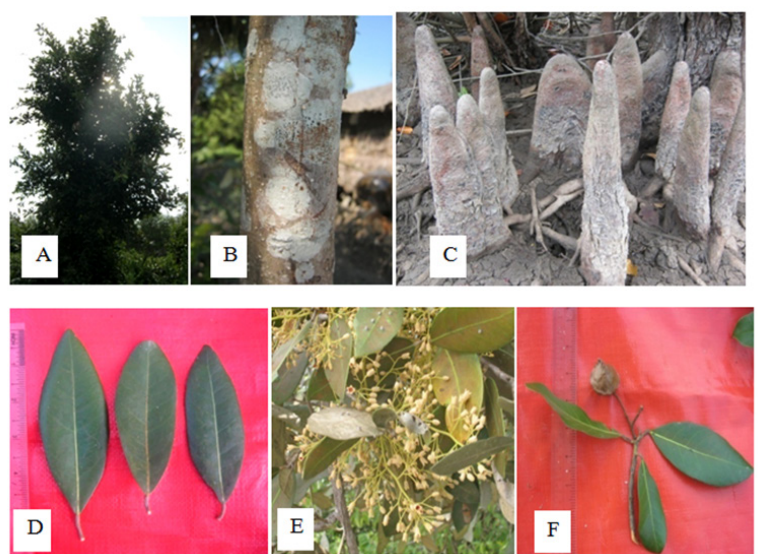

Figure 19 Heritiera fomes: (A) Habit. (B) Stem. (C) Pneumatophores. (D) Leaves. (E) Inflorescence and (F) Fruit as seen.

Flowering and fruting periods- May to September

\section{Discussion}

Myanmar has more than 2,000kilometres of coastline along the Bay of Bengal. Generally, it comprises the Rakhine, Ayeyarwady Delta and Tanintharyi Region, where mangroves are common. Ayeyarwady Delta is in the southern part of Myanmar. It is located between latitudes $15^{\circ}$ and $18^{\circ}$ north, and between longitudes $94^{\circ}$ and $96^{\circ}$ east. The total area of Ayeyarwady Region (including the whole Ayeyarwady Delta) is approximately $155,795 \mathrm{~km}^{2}$. The mangrove of Ayeyarwady Delta is the largest having 180,826 ha, compared to other areas of Rakhine (64,777 ha) and Tanintharyi (140,485 ha) in Myanmar. In Ayeyarwady Delta, mangrove forests are mainly found in Bogalay and Laputta Townships, which are situated at southern part of Ayeyarwady Delta. ${ }^{18}$

According to San Tha Tun, ${ }^{26}$ a total of 41 species of mangroves includes trees, shrubs, herbs, lianas, palms and fern were recorded along the U-To tidal creek, Chaung Tha, western part of Ayeyarwady Division. He reported that Rhizophora apiculata, R. mucronata, Bruguiera gymnorhiza, and Ceriops decandra were common and Bruguiera sexangula, B. parviflora and Ceriops tagal were rare in Chaung Tha area, western part of Ayeyarwady Region. In construct to this data, Bruguiera sexangula species was abundant in the present study area but this species is very rare in Chaung Tha. Likewise, Ceriops decandra was very rare in the present study area but this species is common in Chaung Tha.

The composition of mangroves plants from the natural mangrove forests near the study area were also recorded. A total of 18 species of mangrove plants were recorded in the present study. The common mangrove species are Bruguiera sexangula, Avicennia officinalis, Ceriops tagal and Excoecaria agallocha in the areas.Among the families, Rhizophoraceae, Rhizophora apiculata, Bruguiera sexangula, B.gymnorhiza and Ceriops tagal are more common and Rhizophora mucronata and Ceriops decandra are very rare in the present study.

\section{Conclusion}

In the present study, mangrove plants have been recorded from the natural mangrove areas of Htaung Gyi Tan, Ah Shey Hpyar and War Gone villages, Pyapon Township, South-eastern Ayeyarwady Delta, in Myanmar. In the present study, Avicennia officinalis, Bruguierasexangula and Excoecaria agallocha are the most common in the natural mangroves of South-eastern Ayeyarwady Delta. Now this mangrove areas are gradually declined because local people are clear cut the mangrove trees for fairwood, charcoal and other uses. They haveto work two opportunities to stand their life, fishing and cutting the trees. Nowadays, government, other NGOs and local people are united to estiblish the restored mangrove areas.

\section{Acknowledgements}

I would like to acknowledge my indebtedness to Dr Khin $\mathrm{Mg}$ Oo, Rector of University of Magway and Dr Soe Win and Dr Than Than Oo, Pro-Rectors of University of Magway for their kind permission to carry out this research. I am deeply grateful to Dr Aye Aye Kyi, Professor of Department of Botany, University of Magway for providing facilities for laboratory apparatus, her permission and understanding me through present work.I would like to acknowledge my deepest respect and indebtedness to supervisor Dr. Htay Aung, (Now, Retired and dead) Rector of Mawlamyine University for his valuable guidance, criticism and suggestion and for his kind permission to carry out this research work.I am indebted to Dr Khin Maung Cho, Professor and Head of Department of Marine Science, Pathein University (Now retired) for his useful advice and critical suggestions. I would also like to acknowledge Dr Myint Myint Cho, Professor, Department of Marine Science, Mawlamyime University for her valuable criticism and advice. I am also dreadful to U Aung Myint, Director of Renewable Energy Association Myanmar (REAM) and U Saw Han Shein, Rector of Mawlamyine University (Retired) for their valuable advices, constructive criticisms and facilities of the books. I also especially want to thank U Kyi Lin, Staff Officer, U Kyaw Zaw, Range Officer, U Tin Bo, U Han Myo Linn, U Tin Maung Htwe, Rangers, U Kyaw Naing, Forest Guard and other staff members, Ministry of Environmental Conservation and Forestry, Pyapon Township, Ayeyarwady Region, for their assistance during my field works. Finally, I would like to express my gratitude to my parentfor their encouragement and understanding to accomplish this research.

\section{Conflicts of interest}

The author declares that there are no conflicts of interest.

\section{References}

1. Mephan RH, Mepham JS. The flora of Tidal forests - a rationalization of the use of the term 'mangrove' S. African J Bot 1985;51(2):77-99.

2. Macnae W. A general account of the fauna and flora of mangrove swamps and forests in the Indo-West-Pacific region. Advances in Marine Biology; 1968;6:73-270.

3. Snedaker SC, Snedaker JG. The mangrove ecosystem: reseaech methods. In: Taxonomic considerations of the mangrove species (F. Blasco), UNESCO. Bungay, UK. 1984. p. 81-90,

4. Tomlinson PB. The Botany of Mangroves. Cambridge University Press, Cambridge, London, New York. 1986; p. 1-414.

5. Wightman GM. Mangroves of the Northern Territory. Conservation Commission of the Northern Territory, Palmerston. 1989.

6. Thom BG. Mangrove ecology-a geomorphological perspectives, In: BF Clough editor. Mangrove Ecosystem in Australia: Structure, Function and Management. Canberra: Austr. Nat Univ Press. 1982. p. 3-17. 
7. Duke NC. Australia's Mangroves: The Authoritative Guide to Australia's Mangrove Plants. University of Queensland, Brisbane, Queensland, Australia. 2006.

8. Bunt JS. Introduction. In: Tropical mangrove ecosystem. AI Robertson, DM Alongi, editors, American Geophysical Union, Washington DC, USA 1992. p. 1-6.

9. Ralf S, Saint-Paul U. Mangroves-Forgotten forests, Natural Resources and Development. 1996;43(44):13-36.

10. Twilley RR, Chen RH, Hargis T. Carbon sinks in mangroves and their implications to carbon budget of tropical coastal ecosystems. Water Air Soil Pollut. 1992;64(2):264-288.

11. Giri C, Ochieng E, Tieszen LL. et al. Status and distribution of mangrove forests of the world using earth observation satellite data. Global Ecology and Biogeography. 2010;20:154-159.

12. MAP (Mangrove Action Project), Mangrove Ecosystems. 1990.

13. Wang M, Huang Z, Shi F, et al. Are vegetated areas of mangroves attractive to juvenile and small fish? The case of Dongzhaigang Bay, Hainan Island, China. Estuarine, Coastal and Shelf Science. 2009;85:208-216.

14. Wang Y, Bonynge G, Nugranad J et al, Remote sensing of mangrove change along the Tanzania coast. Marine Geodesy. 2003;26:1-14.

15. Hening RL. Working plan of Sundarbans government forest, Khulna and 24-Parganas district. Bengal, Calcutta. Bengal Secretariate Press. 1892

16. Karim A. Vegetation. In: Mangroves of the Sundarbans. Volume two: Bangladesh. Edited by Hussain Z, G. Acharya. IUCN, Bangkok, Thailand. 1994;43-75.

17. Santisuk, T Taxonomy of the terrestrial trees and shrubs in the mangrove formation in Thailand. Paper presented in the First UNDP/UNESCO Regional Training Course on Introduction to Mangrove Ecosystem, held from 2-30 March, 1983 at NATIONAL Research Council, Bangkok, Thailand. 1983

18. Nay Win Oo Dr. Changes in habitat conditions and conservation of mangrove ecosystem in Myanmar: A case study of Pyindaye Forest reserve, Ayeyarwady Delta. Status Report for MAB Young Scientist Award. 2004; p. $1-15$.
19. Than Htay and Saw Han. Mangrove forest of Burma. In; E Soepadma, editor. "Proceeding of the Asian Symposium on Mangrove Environment Research and Management", University of Malaysia, Kuala Lumpur, Malaysia.1980. 82-85.

20. Aung Myint, Kyaw Soe. Mangrove communities of the Irrawaddy and Salween Deltas of Burma. Wallaceana A Gloval Newsletter for Tropical Ecology. 1985;40:3-5.

21. Cherry Aung, Ma. Study on the mangrove community of Setse coastal area. M. Sc. Thesis. University of Mawlamyine. 1999.

22. San Tha Tun. General accounts on mangrove of Myanmar, University of Pathein', send to Mark D. Spalding. 2007.

23. Tin Hla, Htun Paw Oo. The mangrove forests of Myanmar. M. F. J 2001;5(3):4-10.

24. Thi Thi Htaik. Ecology of mangrove forest in the coastal zone of Mon State with special reference to the family Sonneratiaceae. Unpublished Ph.D Dissertation. Department of Marine Science, Mawlamyine University, Mawlamyine, Myanmar. 2009.

25. Mar Lar. Morphology and ecology of mangroves and associate in Myeik coastal zone. Unpublished Ph.D Dissertation. Department of Botany, Yangon University, Yangon, Myanmar. 2009.

26. San Tha Tun. Species composition and structure of mangrove community along the U-To tidal creek in Chaung Tha. Unpublished Ph.D Dissertation. Department of Marine Science, Mawlamyine University, Mawlamyine, Myanmar. 2011.

27. Nyo Nyo San, Ma. Study on the mangrove plant of Kalagoke Island, Ye Township, Mon State. M. Res (Thesis). Marine Science. Mawlamyine University. 2011.

28. Anonymous. Collected Reports from the 3rd Workshop on "All India Coordinated Research Project on Brackishwater Prawn and Fish Farming", ICAR, New Delhi, India. 1978.

29. Hamilton LS, Snedaker SC. Handbook for Mangrove Area Management. IUCN/UNESCO/UNEP. East-West Centre, Honolulu, Hawaii. 1984. 